\title{
Role of Multidetector Computed Tomography (MDCT) with MPR in Assessment of Fracture Patterns Around the Wrist Joint
}

\author{
Mohammed M. Amin*, Moustafa A. Abdel Wahab*, \\ Ahmed F. Sadek**, Basma M. Abdo Gomaa* \\ * Department of Radiology, Faculty of Medicine, Minia University, Egypt \\ **Department of Orthopaedic Surgery and Traumatology, Faculty of Medicine, Minia University, Egypt
}

\begin{abstract}
Background: Imaging of the wrist is challenging to both radiologists and orthopedic surgeons. This is primarily because of the complex anatomy/functionality of the wrist and also the fact that many frequent injuries are sustained to the hands. Ongoing developments in multidetector computed tomography (MDCT) technology with its "state of the art" postprocessing capabilities have revolution-nized this field. This article pictorially reviews the current clinical role of MDCT imaging of wrist in our practice. Objective: The aim of this work is to study the role of multidetector computed tomography (MDCT) with multi-planar reconstruction in the assessment of fracture patterns around the wrist joint. Patients and methods: Forty patients with fractures around the wrist (diagnosed both clinically and radiologically through plain radiography in the ER) were referred from the orthopedic surgery department for CT evaluation. The study was conducted in the Radiology department at Minia University Hospital during the period from May to December 2018. All patients were enrolled after meeting the inclusion criteria and after having individually written informed consent. The standard CT protocol performed for all patients was as follows: By using 16 slice MDCT machines. Imaging the wrist with the following parameters: $0.625-\mathrm{mm}$ collimation, a pitch of 0.5625 , $120 \mathrm{kVp}$, and $200 \mathrm{~mA} .512 \times 512$ array field-of-view (FOV), and 1 pitch factor. No patient preparation was needed as no contrast media was indicated in the study. Removal of metallic objects was a routine procedure to avoid metallic artifacts. Results: most of the patients were below the age of 37 years, $67.5 \%$ of the studied patients were males and the left side is more affected than the right side. The most common cause of fractures around the wrist is falling on outstretched hand which was statistically superior to other mechanisms (75\%). Radiography and CT detect all cases of distal radius fractures while in ulna fractures radiography sensitivity to fracture was $88.2 \%$, in scaphoid fractures sensitivity was $50 \%$ while in capitate fracture radiography cannot detect fracture $0 \%$ sensitivity. So, CT has the upper hand in the detection of occult fractures. Conclusion: Wrist fractures and dislocations may be overlooked on conventional radiographs. CT with multiplanar and volumetric reformation can be a useful technique to demonstrate occult carpal bone fractures. Also, it can illustrate the complexity and extent of fractures as well as joint dislocations.
\end{abstract}

Key Words: Wrist fractures, MDCT, distal radius fractures, FOOSH, AO classification

\section{Introduction}

Wrist joint is one of the most complex joints in the human body. It is one of the joints that is frequently subjected to injury and results in a considerable functional deficit in daily activities. So, it is essential to precisely diagnose and rapidly manage injuries of the wrist. ${ }^{(1,2)}$ Imaging of the wrist is challenging to both radiologists and orthopedic surgeons. This could be primarily owed to the complex anatomy/ functionality of the wrist in addition to the frequent injuries sustained to the hands. Wrist joint anatomy can be divided into three major partitions: The distal radioulnar, the radiocarpal, and the mid-carpal joints. ${ }^{(1,2)}$ Conventional radiography is the first method of choice in diagnosing wrist traumatic lesions. Eight different shaped bones and a complex threedimensional relationship with each other in the wrist region may be becloud to detect some occult fractures by using conventional radiography. Superposition of anatomical structures, suboptimal positioning and technique, and absent and/or suboptimal patient cooperation in 
emergency settings are factors that may also limit plain radiography role. ${ }^{(3,4,5)}$ With recent development of computed tomography (CT) technologies, multiplanar reformation capability of multidetector computed tomo-graphy (MDCT) technology with its "state of the art" post-processing capabilities and thin slices provide excellent resolution to depict occult bone fractures in skeletal system. ${ }^{(3,4,5)}$ Highresolution MDCT is currently playing a pivotal role in assessing patients following trauma. It is a reference standard as radiography had limited sensitivity in detection of carpal fractures. ${ }^{(6,7)}$ Distal radius fractures which are coined to the term (pilon radiale), are the most common upper extremity fractures constituting $17-18 \%$ of all emergency fractures. Distal radius fractures are usually associated with other bony or soft tissue injuries in variable percentages according to the magnitude of trauma and the bone quality. It shows trimodal pattern of occurrence being common at young adults, after 60 years and in postmenopausal osteoporotic women. ${ }^{(8)}$ Ideal fracture classification should facilitate diagnosis, guide decision making, standardize treatment, help define expected outcomes, and serve as a research tool. MDCT and three-dimensional CT (3D CT) are sometimes required to delineate the fracture lines of complex comminuted intraarticular types. This modality provided great help to the upper limb surgeon as a tool for preoperative planning. ${ }^{(9,10)}$

\section{Aim of the work}

Evaluation of the role of multidetector computed tomography (MDCT) with multiplanar reconstruction in assessment of fracture patterns around the wrist joint.

\section{Patients and Methods}

Forty patients with fractures around the wrist (diagnosed both clinically and radiologically through plain radiography in the ER) who were referred from the orthopedic surgery department for CT evaluation were recruited for a prospective study. The study was conducted in the Radiology department at Minia University Hospital during the period from May to December 2018. All patients were enrolled after meeting the inclusion criteria and after having individually written informed consent. All patients enrolled in this study underwent: Full history taking, thorough Clinical examination, imaging procedures: All Patients underwent radiographically and MDCT evaluation of the wrist. The standard CT protocol performed for all patients was as follows: By using a 16 slice MDCT machine (GE Bright Speed, GE healthcare, Waukesha, WI, and Toshiba Aquilion S16 CFX). Imaging the wrist with the following parameters: $0.625-\mathrm{mm}$ collimation, a pitch of $0.5625,120 \mathrm{kVp}$, and $200 \mathrm{~mA} .512 x 512$ array field-of-view (FOV), and 1 pitch factor.

\section{Results}

The study revealed that the age of the studied patients ranged from 19 to 65 years with the mean age being $37.4 \pm 12.3$ years. There were 23 patients below 37 years $(57.5 \%)$ and 17 of them were above 37 year (42.5\%). Table (1) regarding the gender of our patients 27 cases $(67.5 \%)$ of the studied patients were males and 13 cases $(32.5 \%)$ were females. So, wrist fractures are significantly more common in males than females. $(P=0.01 *)$. Table (2) Regarding the causes of trauma we found that 30 cases were due to fall on outstretched hand (FOOSH) $(75 \%)$ and 10 cases were due to motor car or motorcycle accidents $(25 \%)$. The most common cause of fractures around the wrist is fallen on outstretched hand which was statistically superior to other mechanisms ( $\left.\mathrm{p}=0.002^{*}\right)$. Table (3) Radiographic and MDCT detection of fractures our study has shown that many carpal bone fractures are radiographically occult and detectable only on CT. The most common fracture pattern in the current study was distal radius $38(95 \%)$ of patients detected at radiography with $100 \%$ sensitivity and ulnar styloid process in $17(42.5 \%)$ of patients which detected at radiography with $88.2 \%$ sensitivity while scaphoid fractures were $4(10 \%)$ of patients with $50 \%$ sensitivity. Only one case of capitate fracture was overlooked on radiography with $0 \%$ sensitivity. Table (4) Classification of distal radius fractures according to Ao classification: AO classification has three basic categories of fracture for all bones (Type Aextra articular; Type B-partial articular; Type C-complete articular), which correspond to bending, shear and axial forces. In our study there are 2 cases of type $23 \mathrm{~A} 2,15$ cases of type $23 \mathrm{~B}, 21$ cases of type $23 \mathrm{C}$. 


\section{Case presentation}

Case (1) A male patient aged 40-year-old was presented with a history of motor car accidents and complained of severe pain and swelling in the right wrist. Evidence of comminuted fracture seen involving the right distal radius with mildly displaced bony fragments out of its contour. The fracture is seen reaching the articular surface of the wrist with disruption of both distal radioulnar and radiocarpal joints. No intra-articular loose bodies. Intact examined the styloid process of ulna and carpal bones. No associated soft tissue swellings. (figure 1) shows right distal radial intra-articular fracture $\mathrm{AO}$ type $23 \mathrm{C}$.

Case (2) A male patient aged 37-year-old was presented with a history of falls on an outstretched hand (FOOSH) and complained of severe right wrist pain. There is a fracture line seen involving the right distal radius with mildly displaced fracture ends and displaced bony fragment out of its contour, the fracture seen extending to the articular surface of wrist joint involving the radiocarpal joint. No intra-articular loose bodies. Intact examined the styloid process of the ulna and intact carpal bones. No associated soft tissue swellings. (Figure 2) shows right distal radius intra-articular fracture AO type $23 \mathrm{~B}$.

Case (3) A female patient aged 54 years old was presented with a history of fall on an outstretched hand (FOOSH) and complained of severe pain in the left wrist. Evidence of displaced fracture of left distal radius with intra-articular extension involving both radiocarpal and radioulnar joints. No intraarticular loose bodies. Ulnar styloid process fracture was seen. Intact examined carpal bones and no associated soft tissue swellings. (Figure 3) shows left distal radius intra-articular fracture AO type 23C.

Case (4) A male patient aged 35 years old was presented with a history of fall on out stretched hand $(\mathrm{FOOSH})$ and complained from severe pain and swelling in the right wrist. Evidence of comminuted fracture of right distal radius with intra-articular extension involving both radiocarpal and radioulnar joints. No intra-articular loose bodies. Normal ulnar styloid process. There is a fracture seen in involving the scaphoid and capitate bones. No associated soft tissue swellings. (Figure 4) shows right distal radius intra-articular fracture and $\mathrm{AO}$ type $23 \mathrm{C}$, in addition to fracture of scaphoid and capitate bones. 


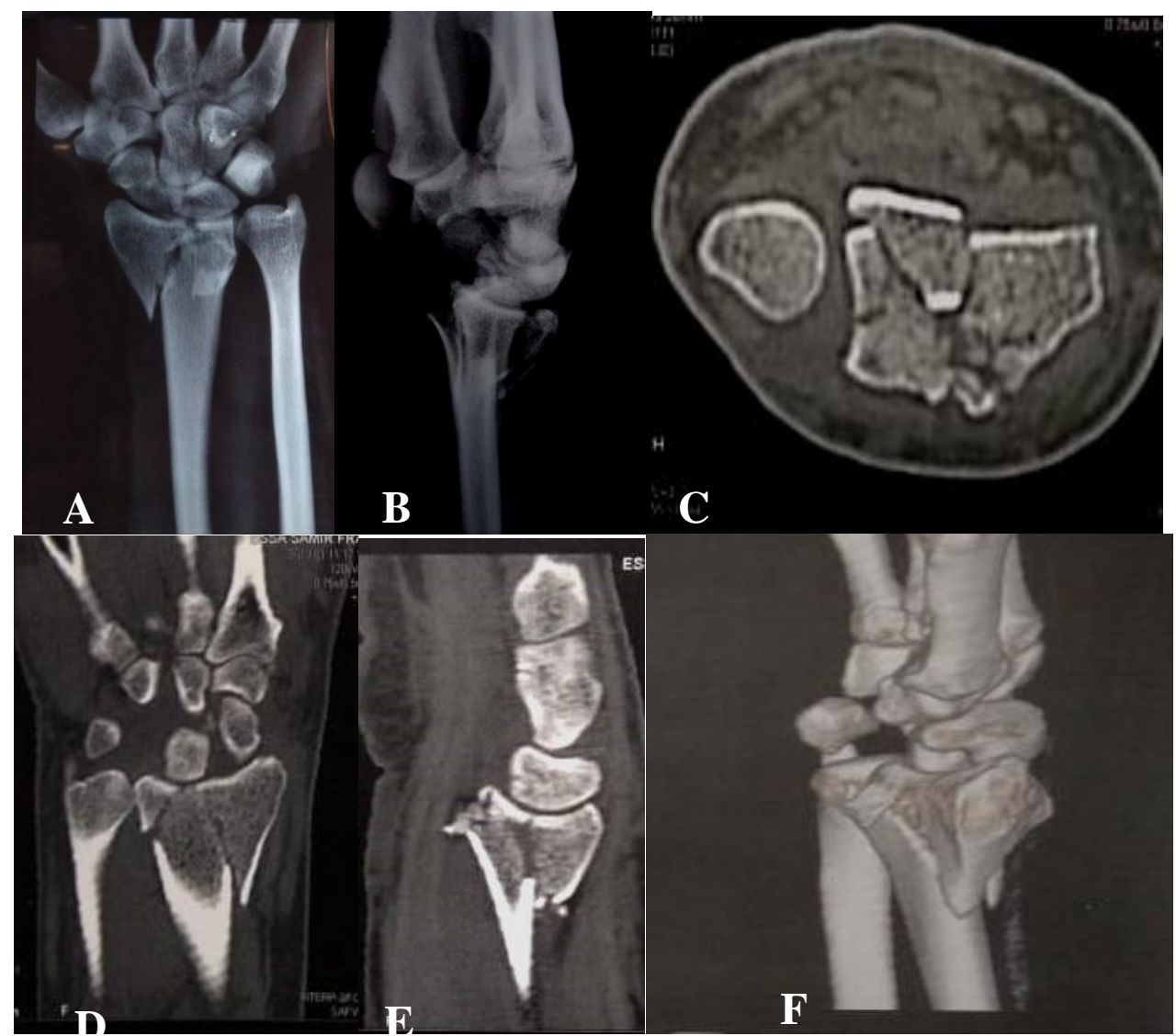

Figure 1: A\&B: X RAY images show displaced fracture of right distal radius. C: Axial image shows comminuted fracture with multiple cortical interruptions. D: Coronal image revealed the disruption of both distal radioulnar and radiocarpal joints. E: Sagittal image proves that the fracture extends to radiocarpal joint. F: $3 D$ image revealed the real extension of the fracture. 


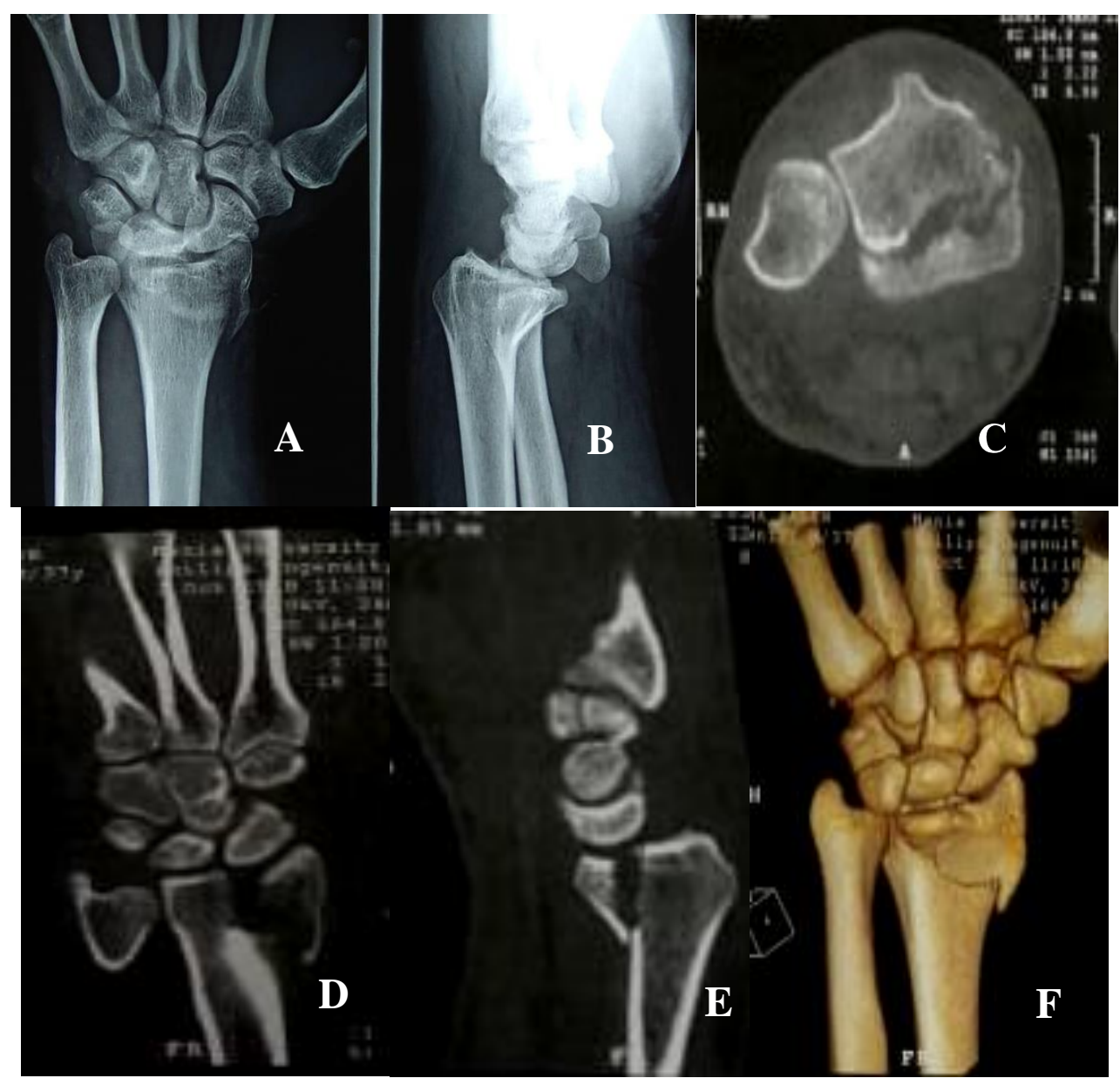

Figure 2: A\&B: X RAY images show displaced fracture seen in right distal radius. $C$ : axial image shows comminuted fracture with normal radioulnar joint. D: coronal image revealed the disruption of radiocarpal joint without interruption of radioulnar joint. E: sagittal image proves that the fracture extend to radiocarpal joint. $F: 3 D$ image revealed the real extension of the fracture. 


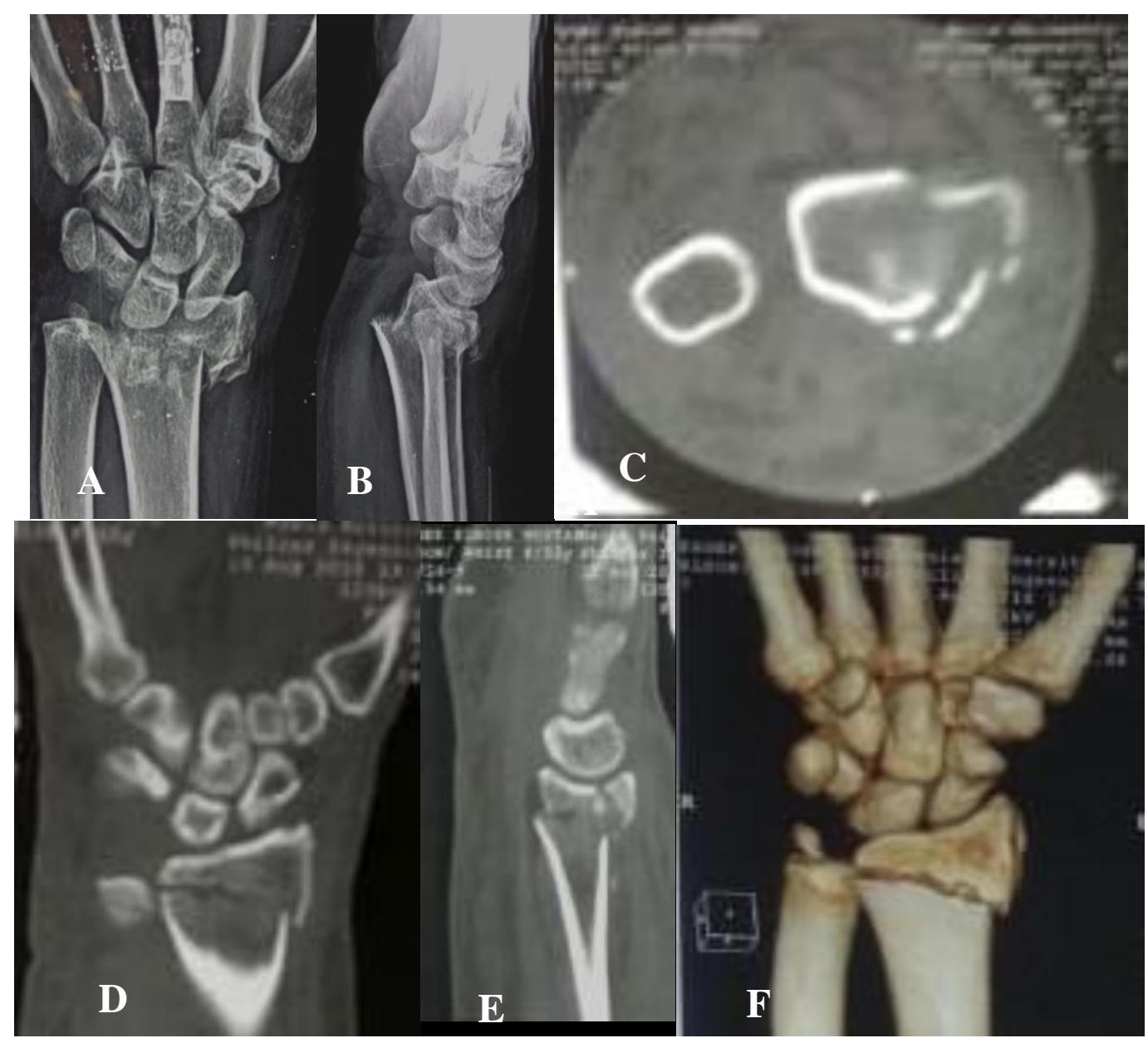

Figure 3: A\&B: X RAY images show displaced fracture of left distal radius with fracture of ulnar styloid process. $C$ : axial image shows comminuted fracture of distal radius. D: coronal image show fracture with disruption of both distal radioulnar and radiocarpal joints with ulnar styloid process fracture. E: sagittal image proves that the fracture extent to radiocarpal joint. $F$ : $3 D$ image revealed the real extension of the fracture. 


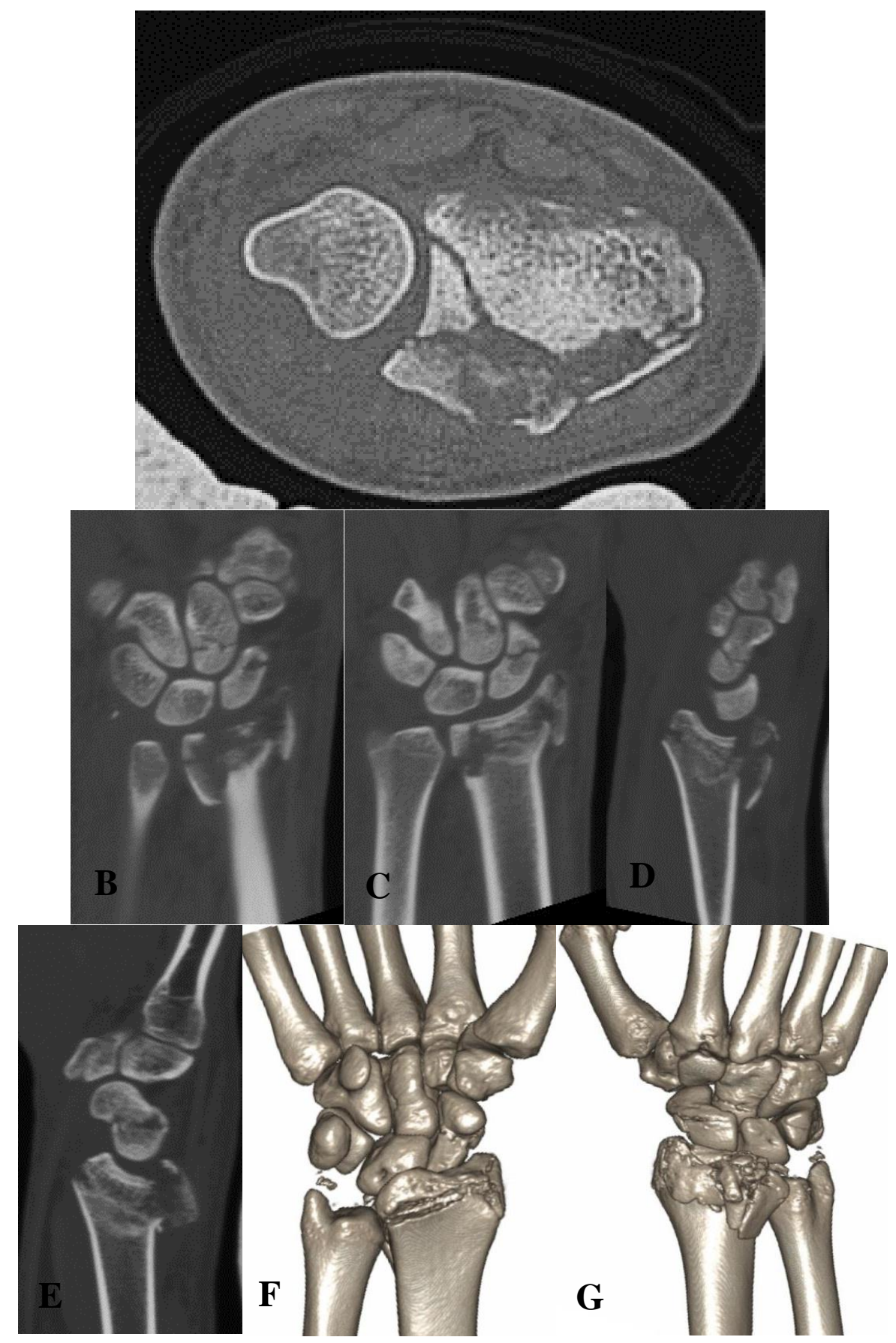

Figure 4: A: axial image show comminuted fracture of distal radius with multiple cortical interruptions. $B \& C$ : coronal images show fracture with disruption of both distal radioulnar and radiocarpal joints, also there are schaphoid and capitate bones fracture. D \& E: sagittal images prove that the fracture reach to radiocarpal joint. $F \& G$ : $3 D$ images revealed the real extension of the fractures.

\section{Discussion}

Regarding the gender of our patients, $67.5 \%$ of the studied patients were males and $32.5 \%$ were females. So that fractures around the wrist are more common in males than females. This is similar to findings in a study done in 2004 by Martti J. Kiuru , et al., where $63.2 \%$ of their patients were males and $36.8 \%$ were females. ${ }^{(7)}$ And also in the study done by C. Vishwanath, et al., 2017 who reported that males were predo- 
minant in their study $(76 \%)^{(11)}$. As well as agree with a study done by P. Jayakumar, et al., 2017 who reported that males were predominant in their study $(85 \%)^{\cdot(12)}$. But this disagrees with welling RD., et al., 2008 who reported that in his study of 60 patients $55 \%$ were females ${ }^{(6)}$ As regards the cause of trauma, the current study revealed that falls on an outstretched hand (FOOSH) were the most common cause of trauma and the motor car or motorcycle accidents were the less common causes. Thirty $(75 \%)$ out of forty patients were due to fall on an outstretched hand and only 10 patients $(25 \%)$ were due to the motor car or motorcycle accidents. So we agree with Y. Karaca, et al., 2016, who found in their results that the usual cause of trauma was FOOSH $(67.1 \%)$ of their patients.(13) But our results contradict C. Vishwanath, et al., 2017 who found that $64 \%$ of their patients were injured in a road traffic accident while only $36 \%$ were injured in falls. ${ }^{(11)}$ After trauma to the rist, radiographic examination often provides valuable diagnostic information for the treating physician ${ }^{(13)}$. However, our study has shown that many carpal bone fractures are radiographically occult and detectable only on CT.

The most common fracture pattern in the current study was distal radius $38(95 \%)$ of patients detected at radiography with $100 \%$ sensitivity and ulanr styloid process in 17 $(42.5 \%)$ of patients which detected at radiography with $88.2 \%$ sensitivity while scaphoid fractures were $4(10 \%)$ of patients with $50 \%$ sensitivity. Only one case of capitate fracture which was overlooked on radiography with $0 \%$ sensitivity. This is in agreement with welling RD., et al., 2008 who reported that radiographic sensitivity for radius fracture was $100 \%$, schaphoid was $81 \%$ while capitate was $0 \%$ while radiographic sensitivity for ulna fracture was $100 \%$ which is slightly different from our study. ${ }^{(6)}$ The current study revealed that distal radius fractures according to $\mathrm{AO}$ classification type $\mathrm{C} 2$ was the most common type of fracture 13 cases (34.2\%). This was in disagreement with P. Kamra, et al., 2019 who found that the usual type of fracture in their study was $\mathrm{AO}$ type $\mathrm{C} 1(32 \%){ }^{(14)}$

\section{Conclusion}

*Conventional radiography is the initial modality available for wrist joint fractures but failed to detect occult fractures around the wrist joint which identified at MDCT.

*MDCT imaging should be performed in patients with a clinically suspected carpal injury even if the plain radiographs do not show any fractures.

*The use of MDCT with MPR and 3D reformatted images allow better visualization of fracture lines, dislocation and comminuted fractures, and optimal evaluation of the injury. *MDCT is useful in preoperative planning and postoperative follow-up of wrist injuries.

\section{References}

1. Syed MA, Raj V and Jeyapalan K. Current role of multidetector computed tomography in imaging of wrist injuries. Curr Probl Diagn Radiol., 2013;42:13-25.

2. Mohammad A, Ahmad A, Ahmed F. Does radiography still have a significant diagnostic role in evaluation of acute traumatic wrist injuries? A prospective comparative study. Emerg radiol., 2018; 25:129-38.

3. Ali B, Işı1 B, Ertuğ YC, et al., Wrist fractures: sensitivity of radiography, prevalence, and patterns in MDCT. Emerg Radiol., 2015;22:251-56.

4. Kaewlai R, Avery LL, Asrani AV, et al., Multidetector CT of carpal injuries: Anatomy, fractures, and fracture-dislocations. Radiographics, 2008; 28:1771-84.

5. Geijer M, El-Khoury GY. MDCT in the evaluation of skeletal trauma: principles, protocols, and clinical applications. Emerg Radiol., 2006;13:7-18.

6. Welling RD, Jacobson JA, Jamadar DA, et al., MDCT and radiography of wrist fractures: radiographic sensitivity and frac-ture patterns. Am J Roentgenol,2008;190:10-16.

7. Kiuru MJ, Haapamaki VV, Koivikko MP, et al., Wrist injuries; diagnosis with multidetector CT. Emerg Radiol.,2004;10: 182-85.

8. Ahmed Fathy MS. Fragment Specific Classification and Fixation of Fractures of the Distal Radius. Int J Sports Sci Med., 2017; 1: 34-43.

9. Mlynarek RA, Lawton JN. Fragmentspecific internal fixation of distal radius fractures. In: Distal radius fractures. Springer international publishing Switzer-land, 2016; 71-87.

10. Medoff R J. Essential radiographic evaluation for distal radius fractures. Hand Clin., 2005; 21: 279-88.

Role of Multidetector Computed Tomography (MDCT) with MPR in Assessment of Fracture 
11. Vishwanath $\mathrm{C}$, Harish K, Gunnaiah KG, et al., Surgical outcome of distal end radius fractures by ligamentotaxis. J Orthop Allied Sci., 2017; 5:68-73.

12. P Jayakumar, T Tunis, B Bravo Giménez. Distal Radius Fracture Classification: Global Perspective on Observer Agreement. J Wrist Surg 2017; 6:46-53.

13. Yunus Karaca, Suha Turkmen, Aysegul Cansu, et al., A study to develop clinical decision rules for the use of radiography in wrist trauma: Karadeniz wrist rules. Am J Emerg Med., 2016; 34: 2074-78.

14. Puneet Kamra, Arun Gulati, Pratik Gopalbhai Patel, et al., Role of external fixator in surgical management of intraarticular distal radius fractures: Functional and radiological outcomes. International Journal of Orthopaedics Sciences. 2019; 5;747-51. 